\title{
The Co-morbidity of Depression and other Chronic Non-communicable Diseases: A review of literature on the epidemiology, diagnosis and health effects
}

\author{
Madeleine Mukeshimana ${ }^{1 *}$ and Gugu Mchunu² \\ ${ }^{1}$ College of Medicine and Health Sciences, University of Rwanda, Kigali, Rwanda \\ ${ }^{2}$ University of KwaZulu Natal, Durban, South Africa
}

\begin{abstract}
Background: Non-communicable diseases are the most common causes of death worldwide. Alongside mortality, noncommunicable diseases also cause high rates of morbidity and disability. The common comorbidity issues of depression worldwide are not a rare occurrence and as depression is chronic in nature it would double or even triple these health threats. Objectives: This paper highlights the epidemiology of the comorbidity of depression as a chronic non-communicable disease, highlighting the health effects of depression and examines specialized tools used to identify depression in patients. Methods: This literature search included the following computerized databases: MEDLINE, Academic Search Premier, Nexus, EBSCOhost, and CINAHL to review articles published from 1991 to 2012. The reviewed articles were quantitative and data were analysed using mostly different versions of SPSS $B$ statistical package. Results and Conclusion: Worldwide established tools were utilized to identify depression in patients with non-communicable diseases. The validated tools include Beck's Depression Inventory, the Patient Health Questionnaire and the Hospital Anxiety and Depression Scale. The prevalence of depression was found to be significantly higher in those with chronic non-communicable diseases in all regions of the world. The health effects of depression found in literature review included a high morbidity and mortality, as well as increased health costs. Conclusion: Significantly higher depression among patients with chronic NCDs suggests that regular screening of depression in this population is warranted. Likewise, the use of validated tool for assessment of depression is essential.
\end{abstract}

Key words: Depression, Non-communicable diseases, comorbidity of depression and non-communicable diseases, detection of comorbidity

\section{Introduction}

The comorbidity of depression as a non-communicable disease (NCD) is becoming an alarming health problem worldwide (Al-Amer, Sobeh, Zayed, \& Al-domi, 2011; Gunn et al., 2012; Katon, 2011). It is against this background that the World Health Organization (WHO) has recommended the regular screening of depression among chronically ill patients (NICE, 2009). However, despite regular screening, depression comorbidity continues to be a serious health problem particularly among those with chronic illnesses. There is a possibility that some cases of depression are missed out due to the fact that some health professionals confuse the signs and symptoms of depression with those of the other co morbid illness such as hypertension, respiratory diseases, diabetes, as well as being more concerned with the physical disorder which is usually the reason for the consultation, and the provider may not be aware of the accompanying depression (Goldberg, 2010). The aim of this paper was to review the epidemiology of the comorbidity of depression and other NCDs, its health effects and tools used to detect depression in non-communicable diseases.

\section{Method}

The literature review method used was an online search using Medline, Academic Search Premier, Nexus, EBSCOhost, and CINAHL conducted using the following key words: depression, non-communicable diseases, comorbidity of depression with non communicable diseases, tools used to detect depression in patients with NCDs. The articles reviewed were those written from 1991 to 2012; studies done before 1991 and after 2012 were excluded. The abstracts were evaluated and were then included in the study if they addressed chronic physical illnesses such as diabetes, hypertension, cancer, and depression as a comorbidity with these physical illnesses. Studies were excluded if: the full-text was not available or was not in English or there was no abstract or the study population was aged below 16 years.

\section{Results}

\section{Epidemiology of the co-morbidity of depression and NCDs}

The prevalence of depression was significantly higher in those with chronic physical illnesses in all regions of the world (Goldberg, 2010). The comorbidity of depression was evidenced in early, as well as recent studies. Examples of early studies which found the coexistence of depression and NCDs includes an Epidemiologic Catchment Area Study (Wells, Golding, \& Burnam, 1989) in the United States of America. The authors found that respondents suffering from one or more of chronic medical conditions had a $41 \%$ increase in the risk of having a psychiatric disorder such as depression, anxiety, or substance abuse. Likewise a large, prospective Canadian community-based study found that there was an increased risk of development of major depression in subjects with chronic medical disorders, compared with those without such disorders; and it was found that a total of $4 \%$ of those with one or more medical condition as compared to $2.8 \%$ of those without medical conditions developed major depression over a 2-year period (Patten, 2001).

Chronic diseases such as cardiovascular diseases, diabetes, cancer and chronic respiratory diseases were associated with higher rates of depression than others. Early studies found that depression occurred in $16 \%$ to $23 \%$ of patients with cardiovascular disease and in almost 1 in 5 patients 
recovering from acute myocardial infarction (Musselman, Evans, \& Nemeroff, 1998; Schleifer \& Macari-Hinson, 1989). Another early controlled study discovered that 35\% of patients with complications of type 2 diabetes mellitus had severe depression as measured by elevated Beck Depression Inventory scores (Leedom, Meehan, Procci, \& Zeidler, 1991). Similarly Lwstman and Clouse (1993) found that individuals with diabetes appear to be at least three times more likely to have depression than the general adult population. Furthermore, a critical review of eight controlled studies found that $14 \%$ of adults with either type 1 or type 2 diabetes mellitus had a major depressive disorder (Lwstman $\&$ Clouse, 1993). It was also noted that approximately $32 \%$ of subjects with diabetes in these controlled studies had clinically significant symptoms of depression.

Recent studies have also confirmed the comorbidity of depression and NCDs. For example the international study of the one year prevalence of depression among 245,400 patients in 60 countries found that patients with two or more chronic physical disorders experienced a prevalence of depression at 23\%, whereas control groups only reported depression in $3.2 \%$ of cases (Moussavi et al., 2007). Studies also found that patients with chronic medical illnesses have been found to have two to three fold increase in the rate of major depression compared with age and gender matched primary care patients (Ali, Stone, Peters, Davies, \& Khunti, 2006; Spijkerman et al., 2005). For example, the prevalence rates of depression in patients with diabetes and coronary heart disease were between $12 \%$ to $18 \%$, and $15 \%$ to $23 \%$ respectively (Ali et al., 2006). Another study found that $80 \%$ of patients with chronic respiratory diseases, screened positive for depression, anxiety, or both (Kunik et al., 2005).

In older patients with Diabetes Mellitus (DM), prevalence of depression ranged from 14 to $17 \%$; in older patients with COPD, prevalence rates of $25 \%$ for minor depression have been reported and prevalence rates of major depression in older COPD patients ranged from 6 to $42 \%$ (Lamers, Jonkers, Bosma, Diederiks, \& van Eijk, 2006). In two reviews done by Stage, Middelboe, Stage and Sorensen (2006) it was found that the prevalence of depression among patients with COPD ranged between $6 \%$ to $57 \%$.

An American study compared one year prevalence of depression in 10,500 patients with chronic disease with 19,460 age matched healthy subjects as controls and found that those subjects suffering from chronic disease were almost three times more likely to be depressed: the odds ratio (OR) was 2.6 (Cl 2.31-2.94). The rates for depression were found to be doubled in diabetes, hypertension, coronary artery disease, and heart failure, and three times higher in those subjects with end stage renal failure, chronic obstructive pulmonary diseases (COPD),and cerebrovascular disease (Egede, 2007). Similarly, the studies done by different authors (Das-Munshi et al., 2007; Katon, 2008) found that people with diabetes overall were twice as likely to have a depression or mixed anxiety/depression than the general population; whereas the figures for those with COPD can four times greater than the general population. Significant panic and depressive symptoms were reported in $16 \%$ to $74 \%$ of patients with Chronic Obstructive Pulmonary Diseases (COPD); unfortunately they were often unrecognized and untreated in primary and speciality care (Motiani, Haidri, \& Rizvi, 2011).

Additionally, numerous studies have found significant depressive disorders in patients with cardiac diseases, cerebrovascular diseases, diabetes and cancers (American Heart Association, 2011; Egede, 2007; Yang et al., 2013).
Depression was found to co-occur in $17 \%$ of cardiovascular cases, $23 \%$ of cerebrovascular cases, $27 \%$ of diabetes patients, and more than $40 \%$ of individuals with cancer (American Heart Association, 2011). Current research shows the 12-month prevalence of major depression to be $9.3 \%$ in individuals with cardiac disease as compared with 4.8\% in those with no comorbid medical illness; depression prevalence ranged from $7.9 \%$ to $17 \%$ in those with other chronic medical conditions (Egede, 2007). The overall prevalence of depression and anxiety were $54.6 \%$ and $49.69 \%$ in Chinese adults with cancer, and the prevalence of depression and anxiety were respectively $18.37 \%$ and $17.50 \%$ in non-cancer group (Yang et al., 2013).

Depression was prevalent among patients who suffered from chronic headaches and musculoskeletal disorders; also researchers found that the lower level of education level and subjects that were younger than forty years of age had associated higher prevalence of depression (Barghouti, Yasein, \& Mustafa, 2013). The American Psychiatric Association recognizes that depression has increased prevalence in persons suffering somatic illness, with prevalence rates being two to three times as high as in persons without somatic illness (American Psychiatric Association, 2010). From the reviewed articles, it is much more evident that the prevalence of depression among patients with different non NCDs is increasing over time.

\section{Health effects of the co-morbidity of depression and NCDs}

Non-compliance to treatment, increase of mortality and morbidity, decrement of quality of life, and the increase of health care costs are some of major health consequences linked to the comorbidity of depression with NCDs. In a meta-analysis of factors related to non-compliance, it was found that depressed patients were three times as likely to be non-compliant with treatment recommendations compared to non-depressed patients (DiMatteo, Lepper, \& Croghan, 2000). In heart disease, Van Melle, De Jonge, Spijkerman, Tijssen, Ormel, Van Veldhuisen et al., (2004) demonstrated a two-fold increase risk of death for those patients with comorbid depression.

Many researchers (Kok et al., 2013; Lamers et al., 2006; Moussavi et al., 2007; Simon, 2001) have found that the comorbidity of depression with NCDs increases the morbidity and mortality, increases health costs, worsens the prognosis for the patient, and reduces the capacity for self-management, which increases the risk of major health complications in patients with NCDs, including high mortality. Simon (2001) compared patients with depression to those without depression, he found that patients with depressive symptoms or disorders experienced decrements in quality of life and had almost twice as many days of restricted activity or missed work because of illness. Simon also discovered that depression was associated with a 50\% to $100 \%$ increase in health services use and costs (Simon, 2001). Simon also found that depression was linked to increased disease as related to morbidity and mortality; in the examination of many population based studies showed a modest association between depression and all-cause mortality and a stronger association between depression and mortality resulting from cardiovascular disease. Similarly, in respective studies by numerous authors (Carney \& Freedland, 2008; Parashar et al., 2006) it was found that major depression and elevated depressive symptoms were associated with a much worse prognosis in patients with Chronic Heart Disease. 
Lamers and colleagues affirmed that chronically ill persons suffering from minor or major depression had an increased mortality risk and a decreased quality of life compared with non-depressed persons with increased health care utilization, medical costs, and disability (Lamers et al., 2006). These studies also showed that depression in fact impairs one's ability to adhere to disease management regimens such as proper diet, exercise, quitting smoking, and taking medication regularly as prescribed which would worsen the course of the chronic illness.

In addition, Kok et al. (2013) affirmed that depression had been associated with poorer outcomes of those with somatic illnesses, in terms of more functional disability, higher health care consumption, and a lower quality of life. Egede (2007) found that the coexistence of major depression, with chronic conditions, was associated with an increase in ambulatory care visits, emergency department visits, days spent in bed because of illness, functional disability, and increased morbidity and mortality.

Depression among patients newly diagnosed with prostate cancer, was associated with higher rates of emergency room visits, hospitalizations, outpatient visits, and an increased risk of death over the course of the follow-up interval. Consequently an increase in health care costs for patients was associated with depression and remained elevated compared with costs for patients without depression, over the course of the follow-up (Jayadevappa, Malkowicz, Chhatre, Johnson, \& Gallo, 2012).

Tools used to detect depression in patients with noncommunicable diseases

\section{Beck Depression Inventory (BDI)}

The Beck Depression Inventory (BDI) is a 21-item; self-report rating inventory that measures characteristic attitudes and symptoms of depression (American Psychiatric Association, 2007). The BDI was originally developed to detect, assess, and monitor changes in depressive symptoms among people in a mental health care setting. The BDI is utilized to detect and quantify depressive symptoms in primary care settings. The BDI can be administered in five and ten minutes as part of a psychological or medical examination (American Psychiatric Association, 2007; Smarr \& Keefer, 2011). The BDI questionnaire has been widely used to screen depression among patients with NCDs such as diabetes and is a well validated tool (Smarr \& Keefer, 2011).

Individual questions of the BDI assess mood, pessimism, and sense of failure, self-dissatisfaction, guilt, punishment, self-dislike, self-accusation, suicidal ideas, crying, irritability, social withdrawal, body image, work difficulties, insomnia, fatigue, appetite, weight loss, bodily preoccupation, and loss of libido. The first Items 1 to 13 are used to assess symptoms that are psychological in nature; while items 14 to 21 assess physical symptomatology. The BDI test is scored differently for the general population and for individuals who have been clinically diagnosed with depression. For the general population, a score of 21 or over represents depression.

\section{Validity and Reliability of BDI}

Validity for BDI has been well documented in literature (American Psychiatric Association, 2007; Smarr \& Keefer. 2011). The BDI has been extensively tested for content validity, concurrent validity, and construct validity. The BDI has content validity, the extent to which items of a test are representative of that which is to be measured. Developed from a consensus among clinicians about depressive symptoms displayed by psychiatric patients, the BDI has concurrent validity and there exists at least 35 studies which have shown concurrent validity between the BDI and other validated measures of depression such as the Hamilton Depression Scale and the Minnesota Multiphasic Personality Inventory-D. Following a range of biological factors, attitudes, and behaviors, tests for construct validity (the degree to which a test measures an internal construct or variable) have shown the BDI to be related to medical symptoms, and psychological stressors such as issues of anxiety, increased stress, loneliness or social isolation, sleep patterns, alcoholism or drug abuse, suicidal behaviors or thoughts, and issues adjustment concerns among youth.

Also studies have confirmed the validity of BDI in a variety of settings using medical populations (e.g., Parkinson's disease, human immunodeficiency virus, oncology, cardiac patients, primary care, chronic pain), persons with disabilities, (e.g., arthritis, spinal cord injury, amputation), medically ill persons of diversity, veterans, students, older adults, adolescents, and many populations with psychiatric diagnoses (e.g., eating disorders, addictions, anxiety disorders) as confirmed by (Smarr \& Keefer, 2011).

The BDI has also been used in different African contexts. Kagee (2008) used it to determine the prevalence rate of depression among patients with chronic illness in South Africa and the scale's internal reliability as measured by Cronbach's alpha was 0.85. Also in their study to explore depression among people living with HIV/AIDS in Rwanda Umubyeyi and Middleton (2010) tested the validity of BDI in Rwandan context and the result of Cronbach's alpha was 0.83.

\section{Reliability}

The reliability of Beck Depression Inventory has been studied and approved. The BDI has been extensively tested for reliability, following established standards for psychological tests published in 1985. The BDI internal consistency has been successfully supported by over 25 studies in many populations; the BDI has been shown to be valid and reliable, with results corresponding to clinician ratings of depression in more than $90 \%$ of all cases (American Psychiatric Association, 2007; Smarr \& Keefer, 2011).

\section{Strengths of BDI}

The BDI was originally developed to provide a quantitative assessment of the intensity of depression. BDI was designed to reflect the depth of depression, and can help monitor mood and depression over time and providing an objective measure for judging improvement, and the effectiveness of the different treatment methods (Steer, Cavalieri, Leonard, $\&$ Beck, 1999).The BDI remains widely used in research; in 1998, it had been utilized in over 2000 empirical studies (Richter et al., 1998). BDI has been translated into multiple European languages as well as Arabic, Chinese, Japanese, Persian and Xhosa (Steele, 2003).

The BDI has also been translated into Kinyarwanda and has been used by different authors to diagnose depression in patients with different chronic diseases (Hagengimana, Hinton, Bird, Pollack, \& Pitman, 2003; Umubyeyi \& Middleton, 2010). BDI is also a common tool used by mental health professionals in Rwanda to diagnose depression. 


\section{Weaknesses of BDI}

The BDI instrument suffers from the same limitations as other self-report inventories, in that scores can be easily exaggerated or minimized by the person completing the BDI. Like all questionnaires, the method that the instrument is administered can have an effect on the final score. If a patient is asked to fill out the form in front of other people in a clinical environment, for instance, social expectations have been shown to elicit a different response compared to administration via a postal survey (Bowling, 2005).

In participants with concomitant physical illness the BDI's reliance on physical symptoms such as fatigue, may be suffer from artificially inflated scores due to symptoms of the illness, rather than of depression (Moore, Moore, \& Shaw, 1998). In an effort to deal decrease this limitation Beck and his colleagues developed the "Beck Depression Inventory for Primary Care" (BDI-PC), a short screening scale consisting of seven items from the BDI-II considered to be independent of physical function. Unlike the standard $\mathrm{BDI}$, the BDI-PC produces only a binary outcome of "not depressed" or "depressed" for patients above a cutoff score of 4 (Steer et al., 1999). Although designed as a screening device rather than a diagnostic tool, the BDI-PC is sometimes used by health care providers to reach a quick diagnosis (Hersen, Turner, \& Beidel, 2011).

\section{The Patient Health Questionnaire (PHQ-9)}

The PATIENT HEALTH QUESTIONNAIRE (PHQ-9) offers psychologists a concise, self-administered tool for assessing depression. The PHQ-9 incorporates the DSMIV depression criteria, with other leading major depressive symptoms, into a brief self-report instrument. The PHQ-9 is commonly used for screening and diagnosis, as well as selecting and monitoring treatment of depression (American Psychiatric Association, 2014).

\section{Validity and reliability of PHQ-9}

The diagnostic validity of the 9-item $\mathrm{PHO}-9$ has been established in studies involving 8 primary care and 7 obstetrical clinics. PHQ-9 scores greater than 10 had a sensitivity of $88 \%$ and a specificity of $88 \%$ for Major Depressive Disorder. Reliability and validity of the PHQ-9 tool showed sound psychometric properties and internal validity of the PHQ-9 has been shown to be high. A study involving two different patient populations produced Cronbach alphas of .86 and .89. Criteria validity was established by conducting 580 structured interviews by mental health professionals. The results from these interviews showed that individuals who scored high $(\geq 10)$ on the PHQ-9 were between 7 to 13.6 times more likely to be diagnosed with depression by the mental health professional. On the other hand, individuals scoring low ( $\leq$ 4) on the PHQ-9 had a less than a 1 in 25 chance of having depression (American Psychiatric Association, 2014).

\section{Strengths of PHQ-9 questionnaire}

The PHQ-9 has been used in many studies in primary care settings to detect depression among patients with NCDs. The PHQ-9 has also been used to screen depression among older individuals who have suffered from physically disabling conditions (American Psychiatric Association, 2014). The questions of the $\mathrm{PHQ}-9$ are easily understood and the other advantage of the instrument included its length and ease of scoring(Perkowski, 2013).

\section{Weaknesses of the PHQ-9 questionnaire}

The PHQ-9 was particularly limited in identifying individuals with anxiety disorders, other than panic disorder. The PHQ9 tool was found to have limitations in identifying depressed individuals with dysthymia (Eack, Greeno, \& Lee, 2006).

A disadvantage of the $\mathrm{PHQ}-9$ is the lack of use and validation in studies within health sciences education. Further research is needed to evaluate the psychometric properties in samples of health science students and practitioners (Perkowski, 2013).

\section{The Hospital Anxiety and Depression Scale (HADS)}

Hospital Anxiety and Depression Scale (HADS) was originally developed by Zigmond and Snaith in1983 and is commonly used by providers to determine the levels of anxiety and depression patients may be experiencing. The HADS is a fourteen item scale that generates ordinal data. Seven of the items related to anxiety and seven items correlate to depressive symptoms (Snaith, 2003). Zigmond and Snaith created this outcome measure specifically to avoid reliance on aspects of these conditions that are common somatic symptoms of illness, such as fatigue and insomnia or hypersomnia. The HADS was created as a tool for the detection of anxiety and depression in people with physical illnesses such as diabetes, cardiac diseases, cancer, respiratory diseases, etc. (Snaith, 2003).

\section{Validity and Reliability of HADS}

Validity: The validity of HADS has been proved by concurrent validity established in a number of studies as confirmed by Snaith (2003)

Reliabity: HADS has been proved to be a reliable tool to detect depression among patients with or without NCDs For example internal consistency described for patients with cancer: Anxiety subscale Cronbach's alpha was 0.93; Depression subscale alpha was 0.9 (Moorey et al., 1991).

In a healthy United Kingdom (UK) sample (Without non communicable diseases), internal consistency for anxiety and depression found the total scores of 0.82, 0.77 and 0.86 respectively (Crawford, Henry, Crombie, \& Taylor, 2001). Test-retest reliability within a healthy sample with a correlation for a depression scale at 0.92; anxiety subscale was found to be 0.89 (Snaith, 2003).

Also In a study done by (Yusoff, Low, \& Yip, 2011), a study of husbands with a breast cancer patients; the psychometric properties of the HADS were reported based on Cronbach' alpha, Intraclass Correlation Coefficients (ICC), Effect Size Index (ESI), sensitivity and discriminity of the scale. Internal consistency of the scale was excellent, with Cronbach's alpha of 0.88 for Anxiety subscale and 0.79 for Depression subscale. Test-retest Intraclass Correlation Coefficient (ICC) was 0.35 and 0.42 for Anxiety and Depression Subscale, respectively.

\section{Strengths of HADS questionnaire}

The HADS is brief and simple to administer, though it was originally designed to be used within the hospital populations, this tool has been found to perform well with non-hospitalized groups (McDowell, 2006). Snaith (2003) found the HADS to take only an average 2-5 minutes to complete and is completed by the patients themselves. The HADS has been found to perform as well as the BDI 
and the PHQ-9 evaluation instruments. Overall the HADS scale possessed good psychometric properties in terms of factor structure, intercorrelation, homogeneity, and internal consistency (Mykletun, Stordal, \& Dahl, 2001).

The strengths of the HADS includes three major points (Mykletun et al., 2001) :

- Interpretability: The results are easy to interpret with higher scores on each individual scale or the entire scale indicating greater anxiety, depression or mood disorders.

- $\quad$ Acceptability: The HADS is widely accepted and used with most patient populations including those with chronic diseases.

- Feasibility: The scale is readily available and can be utilized free of charge. The HADS takes only a few minutes to complete and no specialized training is need to administer the test and may be completed by the patients themselves.

\section{Weaknesses of HADS questionnaire}

Limitations HADS questionnaire in the diagnosis of depression or depressive symptoms associated with chronic illnesses, the symptoms of chronic illnesses may confound the test scores (Whelan-Goodinson, Ponsford, \& Schönberger, 2009). Dawkins, Cloherty, Gracey and Evans (2006) stated that caution is recommended when interpreting the results of these scales.

\section{Conclusion}

The relationship between depression and NCDs is research is well supported in literature. The prevalence of depression in patient with NCDs is increasing worldwide. Published reports described increased morbidity and mortality, reduced quality of health, increased health costs related to the co-morbidity of depression with NCDs. Different tools exist to detect depression in patients with NCDs including the Beck Depression Inventory (BDI), the Hospital Anxiety and Depression Scale (HADS), and the Patient Health Questionnaire (PHQ-9). The clinical relevance of this theme is clear, since depression is associated with NCDs, depression should be regularly screened for in patients with NCDs, and adequate treatment should be administered to patients with the co-morbidity of depression and anxiety issues.

The authors recommend future research about exploration of prevalence of depression in patients with NCDs in Rwanda; exploration of management of this co-morbidity in Rwandan context and developing a protocol to manage this co morbidity in Rwanda.

\section{Acknowledgements}

Our deep appreciation and thanks go to the Rwanda Education Board for financial support. We also express our deep gratitude to Professor Njunwa Kato for his valuable support and guidance.

\section{Conflict of interest}

All authors report no conflict of interest.

\section{Authors' contributions}

MM developed the concept for the comprehensive literature review; GM reviewed research and assisted with substantial edits. All authors approve the final version.

\section{References}

Al-Amer, R. M., Sobeh, M. M., Zayed, A. A., \& Al-domi, H. A. (2011). Depression among adults with diabetes in Jordan: risk factors and relationship to blood sugar control. Journal of Diabetes and its Complications, 25(4), 247-252.

Ali, S., Stone, M., Peters, J., Davies, M., \& Khunti, K. (2006). The prevalence of co-morbid depression in adults with Type 2 diabetes: a systematic review and meta-analysis. Diabetic Medicine, 23(11), 1165-1173.

American Heart Association. (2011). Depression and Heart Health. Retrieved 21st September 2012, from http://www.heart.org/HEARTORG/ Conditions/More/MyHeartandStrokeNews/ Depression-and-Heart-Health_UCM_440444_ Article.jsp.

American Psychiatric Association. (2007). Practice guideline for the treatment of patients with major depressive disorder: American Psychiatric Association.

American Psychiatric Association. (2010). Practice guideline for the treatment of patients with major depressive disorder (3 ed.). Washington,DC: American Psychiatric Association.

American Psychiatric Association. (2014). Patient Health Questionnaire (PHQ-9 \& PHQ-2). Retrieved September 15, 2014 http://www.apa.org/pi/ about/publications/caregivers/practice-settings/ assessment/tools/patient-health.aspx

Barghouti, F., Yasein, N. A., \& Mustafa, R. A. B. (2013). Depression and co-morbid chronic illnesses in family practice. HealthMED, 7(2), 477.

Bowling, A. (2005). Mode of questionnaire administration can have serious effects on data quality. Journal of public health, 27(3), 281-291.

Carney, R. M., \& Freedland, K. E. (2008). Depression in patients with coronary heart disease. The American journal of medicine, 121(11), S20-S27.

Crawford, J., Henry, J., Crombie, C., \& Taylor, E. (2001). Normative data for the HADS from a large non-clinical sample. British Journal of Clinical Psychology, 40(4), 429-434.

Das-Munshi, J., Stewart, R., Ismail, K., Bebbington, P. E., Jenkins, R., \& Prince, M. J. (2007). Diabetes, common mental disorders, and disability: findings from the UK National Psychiatric Morbidity Survey. Psychosomatic Medicine, 69(6), 543-550. 
Dawkins, N., Cloherty, M. E., Gracey, F., \& Evans, J. J. (2006). The factor structure of the Hospital Anxiety and Depression Scale in acquired brain injury. Brain injury, 20(12), 1235-1239.

DiMatteo, M. R., Lepper, H. S., \& Croghan, T. W. (2000). Depression is a risk factor for noncompliance with medical treatment: meta-analysis of the effects of anxiety and depression on patient adherence. Archives of Internal Medicine, 160(14), 2101-2107.

Eack, S. M., Greeno, C. G., \& Lee, B.-J. (2006). Limitations of the Patient Health Questionnaire in identifying anxiety and depression in community mental health: many cases are undetected. Research on social work practice, 16(6), 625-631.

Egede, L. E. (2007). Major depression in individuals with chronic medical disorders: prevalence, correlates and association with health resource utilization, lost productivity and functional disability. General hospital psychiatry, 29(5), 409-416.

Goldberg, D. (2010). The detection and treatment of depression in the physically ill. World Psychiatry, 9(1), 16-20.

Gunn, J. M., Ayton, D. R., Densley, K., Pallant, J. F., Chondros, P., Herrman, H. E., \& Dowrick, C. F. (2012). The association between chronic illness, multimorbidity and depressive symptoms in an Australian primary care cohort. Social psychiatry and psychiatric epidemiology, 47(2), 175-184.

Hagengimana, A., Hinton, D., Bird, B., Pollack, M., \& Pitman, R. K. (2003). Somatic panic-attack equivalents in a community sample of Rwandan widows who survived the 1994 genocide. Psychiatry research, 117(1), 1-9.

Hersen, M., Turner, S. M., \& Beidel, D. C. (2011). Adult psychopathology and diagnosis: John Wiley \& Sons.

Jayadevappa, R., Malkowicz, S. B., Chhatre, S., Johnson, J. C., \& Gallo, J. J. (2012). The burden of depression in prostate cancer. Psycho-Oncology, 21(12), 13381345.

Kagee, A. (2008). Symptoms of depression and anxiety among a sample of South African patients living with a chronic illness. Journal of Health Psychology, 13(4), 547-555.

Katon, W. (2008). The comorbidity of diabetes mellitus and depression. The American journal of medicine, 121(11), S8-S15.

Katon, W. (2011). Epidemiology and treatment of depression in patients with chronic medical illness. Dialogues in clinical neuroscience, 13(1), 7.

Kok, G. D., Bockting, C. L., Burger, H., Hannig, W., Pijnenborg, G. H., Cuijpers, P., \& Hollon, S. D. (2013). Double trouble: does co-morbid chronic somatic illness increase risk for recurrence in depression? A systematic review. PloS one, 8(3), e57510.
Kunik, M. E., Roundy, K., Veazey, C., Souchek, J., Richardson, P., Wray, N. P., \& Stanley, M. A. (2005). Surprisingly high prevalence of anxiety and depression in chronic breathing disorders. Chest Journal, 127(4), 12051211.

Lamers, F., Jonkers, C., Bosma, H., Diederiks, J., \& van Eijk, J. (2006). Effectiveness and cost-effectiveness of a minimal psychological intervention to reduce non-severe depression in chronically ill elderly patients: the design of a randomised controlled trial [ISRCTN92331982]. BMC Public Health, 6(1), 161.

Leedom, L., Meehan, W. P., Procci, W., \& Zeidler, A. (1991). Symptoms of depression in patients with type II diabetes mellitus. Psychosomatics, 32(3), 280-286.

Lwstman, P., \& Clouse, R. (1993). Prevalence of depression in adults with diabetes. Diabetes care, 16(8), 11671178.

McDowell, I. (2006). Measuring health: a guide to rating scales and questionnaires: Oxford University Press.

Moore, M. J., Moore, P. B., \& Shaw, P. J. (1998). Mood disturbances in motor neurone disease. Journal of the neurological sciences, 160, S53-S56.

Moorey, S., Greer, S., Watson, M., Gorman, C., Rowden, L., Tunmore, R., Bliss, J. (1991). The factor structure and factor stability of the hospital anxiety and depression scale in patients with cancer. The British Journal of Psychiatry, 158(2), 255-259.

Motiani, B., Haidri, F. R., \& Rizvi, N. (2011). Frequency of depression in Chronic Obstructive Pulmonary Disease (COPD) patients. Pakistan Journal of Medical Sciences, 27(5).

Moussavi, S., Chatterji, S., Verdes, E., Tandon, A., Patel, V., \& Ustun, B. (2007). Depression, chronic diseases, and decrements in health: results from the World Health Surveys. The Lancet, 370(9590), 851-858.

Musselman, D. L., Evans, D. L., \& Nemeroff, C. B. (1998). The relationship of depression to cardiovascular disease: epidemiology, biology, and treatment. Archives of general psychiatry, 55(7), 580-592.

Mykletun, A., Stordal, E., \& Dahl, A. A. (2001). HospitalAnxiety and Depression (HAD) scale: factor structure, item analyses and internal consistency in a large population. The British Journal of Psychiatry, 179(6), 540-544.

NICE. (2009). Depression in adults: The treatment and management of depression in adults. NICE guideliine, 90.

Parashar, S., Rumsfeld, J. S., Spertus, J. A., Reid, K. J., Wenger, N. K., Krumholz, H. M., Dawood, N. (2006). Time course of depression and outcome of myocardial infarction. Archives of Internal Medicine, 166(18), 2035-2043.

Patten, S. B. (2001). Long-term medical conditions and major depression in a Canadian population study at waves 1 and 2. Journal of affective disorders, 63(1), 35-41. 
Perkowski, L. (2013). Critical Synthesis Package: Patient Health Questionnaire (PHQ-9): MedEdPORTAL Publications.

Richter, P., Werner, J., Heerlein, A., s, e., Kraus, A., \& Sauer, H. (1998). On the validity of the Beck Depression Inventory. Psychopathology, 31(3), 160-168.

Schleifer, S. J., \& Macari-Hinson, M. M. (1989). The nature and course of depression following myocardial infarction. Archives of Internal Medicine, 149(8), 1785-1789.

Simon, G. E. (2001). Treating depression in patients with chronic disease. Western Journal of medicine, 175(5), 292.

Smarr, K. L., \& Keefer, A. L. (2011). Measures of depression and depressive symptoms: Beck Depression Inventory-II (BDI-II), Center for Epidemiologic Studies Depression Scale (CES-D), Geriatric Depression Scale (GDS), Hospital Anxiety and Depression Scale (HADS), and Patient Health Questionnaire-9 (PHQ-9). Arthritis care \& research, 63(S11), S454-S466.

Snaith, R. P. (2003). The hospital anxiety and depression scale. Health Qual life outcomes, 1(1), 29.

Spijkerman, T., de Jonge, P., van den Brink, R. H., Jansen, J. H., May, J. F., Crijns, H. J., \& Ormel, J. (2005). Depression following myocardial infarction: firstever versus ongoing and recurrent episodes. General hospital psychiatry, 27(6), 411-417.

Stage, K. B., Middelboe, T., Stage, T. B., \& Sørensen, C. H. (2006). Depression in COPD-management and quality of life considerations. International journal of chronic obstructive pulmonary disease, 1(3), 315.

Steele, G. I. (2003). The development and validation of the Xhosa translations of the Beck Depression Inventory, the Beck Anxiety Inventory, and the Beck Hopelessness Scale. Department of Psychology, Rhodes University, Grahamstown.

Steer, R. A., Cavalieri, T. A., Leonard, D. M., \& Beck, A. T. (1999). Use of the Beck Depression Inventory for Primary Care to screen for major depression disorders. General hospital psychiatry, 21(2), 106-111.

Umubyeyi, B., \& Middleton, L. (2010). Exploring depression among people living with HIV/AIDS and attending a primary health care centre in Kigali-Rwanda: a descriptive, cross-sectional study. Retrieved 1 March, 2016, from http://researchspace.ukzn.ac.zal xmlui/bitstream/handle/10413/893/Benoite_U_2010. pdf? sequence $=1$

Van Melle, J. P., De Jonge, P., Spijkerman, T. A., Tijssen, J. G., Ormel, J., Van Veldhuisen, D. J., Van Den Berg, M. P. (2004). Prognostic association of depression following myocardial infarction with mortality and cardiovascular events: a meta-analysis. Psychosomatic Medicine, 66(6), 814-822.
Wells, K. B., Golding, J. M., \& Burnam, M. A. (1989). Affective, substance use, and anxiety disorders in persons with arthritis, diabetes, heart disease, high blood pressure, or chronic lung conditions. General hospital psychiatry, 11(5), 320-327.

Whelan-Goodinson, R., Ponsford, J., \& Schönberger, M. (2009). Validity of the Hospital Anxiety and Depression Scale to assess depression and anxiety following traumatic brain injury as compared with the Structured Clinical Interview for DSM-IV. Journal of affective disorders, 114(1), 94-102.

Yang, Y.-L., Liu, L., Wang, Y., Wu, H., Yang, X.-S., Wang, J.-N., $\&$ Wang, L. (2013). The prevalence of depression and anxiety among Chinese adults with cancer: a systematic review and meta-analysis. BMC cancer, 13(1), 393

Yusoff, N., Low, W. Y., \& Yip, C.-H. (2011). Psychometric properties of the Malay Version of the hospital anxiety and depression scale: a study of husbands of breast cancer patients in Kuala Lumpur, Malaysia. Asian Pac J Cancer Prev, 12(4), 915-917. 